\title{
Solution Procedure for a Problem of Dynamical Optimization with Control Delays *
}

\author{
Mikhail Gomoyunov* \\ * Institute of Mathematics and Computer Sciences, \\ Ural Federal University, Mira str. 19, Ekaterinburg, 620002, Russia \\ (e-mail:m.i.gomoyunov@gmail.com).
}

\begin{abstract}
In this paper a linear dynamical system controlled under the conditions of disturbances and with control delays is considered. The Euclidean norm of a set of the system motion deviations at given instants of time from the origin is minimized. A typical case of the location of the indicated instants of time is studied. Within the game-theoretical approach the problem of calculating the value of the optimal guaranteed result and constructing a positional (closed-loop) control law that ensures this result is posed. For this problem, an effective solution procedure based on the recurrent construction of upper convex hulls of auxiliary program functions is elaborated. Results of numerical simulations are given.
\end{abstract}

Keywords: control theory; differential games; time-delay.

\section{INTRODUCTION}

In this paper a linear dynamical system controlled under the conditions of disturbances is considered. The goal of the control is to minimize the Euclidean norm of a set of the system motion deviations at given instants of time from the origin. Within the game-theoretical approach of Krasovskii and Subbotin (1988) (see also Osipov and Pimenov (1981); Krasovskii (1985); Krasovskii and Krasovskii (1995)) the problem of calculating the value of the optimal guaranteed result and constructing a positional (closed-loop) control law that ensures this result is posed.

In Gomoyunov and Lukoyanov (2012) this problem was reduced to the calculation of the game value and the construction of the minimax strategy in an auxiliary zerosum differential game without control delays and with the terminal cost function. For calculating the game value the upper convex hulls method (see, e.g., Krasovskii and Krasovskii (1995)) was applied. For constructing the minimax strategy the method of extremal shift to the accompanying point (see, e.g., Krasovskii (1985)) was used. As a result, for the initial problem, a solution procedure was obtained. It is based on the recurrent construction of upper convex hulls of auxiliary functions, which are defined on the domain, whose dimension depends on the number $N$ of instants of time at which the motion quality is evaluated. So this dimension can be large even if the dimension of the state vector of the dynamical system is small. This dependency narrows the application field of the procedure.

\footnotetext{
* This work is supported by the Program of the Presidium of the Russian Academy of Sciences "Dynamical Systems and Control Theory" (12-P-1-1002) and by the Russian Foundation for Basic Research (12-01-00290).
}

In the present paper, a new solution procedure is proposed for some particular but typical case of the location of the instants at which the motion quality is evaluated. In this new procedure the dimension of the domains of the auxiliary functions being convexified becomes significantly less and independent of $N$.

The paper develops the constructions from Lukoyanov (1998) for the case of systems with control delays (see, e.g., Banks et al (1971); Osipov and Pimenov (1981)).

\section{STATEMENT OF THE PROBLEM}

Consider a dynamical system described by the following differential equation

$$
\begin{gathered}
\frac{\mathrm{d} x(t)}{\mathrm{d} t}=A(t) x(t)+B(t) u(t)+B_{\tau}(t) u(t-\tau)+C(t) v(t), \\
t_{0} \leq t \leq \vartheta, \quad x \in \mathbb{R}^{n}, \quad u \in P \subset \mathbb{R}^{r}, \quad v \in Q \subset \mathbb{R}^{s} .
\end{gathered}
$$

and the initial condition

$$
\begin{gathered}
x\left(t_{0}\right)=x_{0} \in \mathbb{R}^{n}, \\
u_{t_{0}}(\cdot)=\left\{u_{t_{0}}(\xi)=u\left(t_{0}+\xi\right), \xi \in[-\tau, 0)\right\}=p_{0}(\cdot) \in \mathcal{P} .
\end{gathered}
$$

Here $t$ is the time variable, $x$ is the state vector, $u$ is the control vector, and $v$ is the vector of unknown disturbances; $t_{0}$ and $\vartheta$ are respectively the initial and the terminal instants of time $\left(t_{0}<\vartheta\right) ; P$ and $Q$ are known compact sets; matrix functions $A(t), B(t), B_{\tau}(t)$ and $C(t)$ are continuous; $\tau=$ const $>0$ is the delay value; $\mathcal{P}$ is the set of all Borel measurable functions from $[-\tau, 0)$ to $P$.

Let $\lambda>0$ be such that

$$
\left\|A(t) x+B(t) u_{1}+B_{\tau}(t) u_{2}+C(t) v\right\| \leq(1+\|x\|) \lambda
$$

for all $t \in\left[t_{0}, \vartheta\right], x \in \mathbb{R}^{n}, u_{1}, u_{2} \in P$ and $v \in Q$. Here and below $\|\cdot\|$ is the Euclidian norm. 
A triple $(t, x, p(\cdot)) \in\left[t_{0}, \vartheta\right] \times \mathbb{R}^{n} \times \mathcal{P}$ is called a position of system (1). The set $K$ of all possible positions is defined as follows:

$$
\begin{aligned}
K=\left\{(t, x, p(\cdot)) \in\left[t_{0}, \vartheta\right]\right. & \times \mathbb{R}^{n} \times \mathcal{P}: \\
\|x\| & \left.\leq\left(1+\left\|x_{0}\right\|\right) e^{\left(t-t_{0}\right) \lambda}-1\right\} .
\end{aligned}
$$

Let $\left(t_{*}, x_{*}, p_{*}(\cdot)\right) \in K, t_{*}<\vartheta$, and $t^{*} \in\left(t_{*}, \vartheta\right]$ be given. We assume that admissible control and disturbance realizations are Borel measurable functions

$$
\begin{aligned}
& u\left[t_{*}[\cdot] t^{*}\right)=\left\{u(t) \in P, t_{*} \leq t<t^{*}\right\}, \\
& v\left[t_{*}[\cdot] t^{*}\right)=\left\{v(t) \in Q, t_{*} \leq t<t^{*}\right\} .
\end{aligned}
$$

In addition, we put

$$
u(t)=p_{*}\left(t-t_{*}\right), \quad t_{*}-\tau \leq t<t_{*} .
$$

From the position $\left(t_{*}, x_{*}, p_{*}(\cdot)\right)$, such realizations $u\left[t_{*}[\cdot] t^{*}\right)$ and $v\left[t_{*}[\cdot] t^{*}\right)$ uniquely generate the system motion

$$
x\left[t_{*}[\cdot] t^{*}\right]=\left\{x(t) \in \mathbb{R}^{n}, t_{*} \leq t \leq t^{*}\right\},
$$

that is an absolutely continuous function, which satisfies the condition $x\left(t_{*}\right)=x_{*}$, and, together with the corresponding $u(t)$ and $v(t)$, satisfies equation (1) for almost all $t \in\left[t_{*}, t^{*}\right]$. Note that, for any $t \in\left[t_{*}, t^{*}\right]$, the inclusion $\left(t, x(t), u_{t}(\cdot)\right) \in K$ is valid, where

$$
u_{t}(\cdot)=\left\{u_{t}(\xi)=u(t+\xi), \xi \in[-\tau, 0)\right\} .
$$

Let $x\left[t_{0}[\cdot] \vartheta\right]$ be the system motion, that has been generated from the initial position $\left(t_{0}, x_{0}, p_{0}(\cdot)\right) \in K$ by some admissible realizations $u\left[t_{0}[\cdot] \vartheta\right)$ and $v\left[t_{0}[\cdot] \vartheta\right)$. The quality of this motion is evaluated by the cost function:

$$
\gamma=\left(\sum_{i=1}^{N}\left\|x\left(\vartheta_{i}\right)\right\|^{2}\right)^{1 / 2}
$$

where $\vartheta_{i}=t_{0}+i \tau, i=\overline{1, N}, \vartheta_{N}=\vartheta$.

The goal of the control is to minimize function (3). Note that, since disturbance actions are unknown, the worstcase may occur when disturbances maximize (3).

A control strategy $U(\cdot)$ is an arbitrary function

$$
U(\cdot)=\{U(t, x, p(\cdot), \varepsilon) \in P,(t, x, p(\cdot)) \in K, \varepsilon>0\},
$$

where $\varepsilon>0$ is the accuracy parameter (see, e.g., Krasovskii (1985); Krasovskii and Krasovskii (1995)).

The strategy $U(\cdot)$ acts onto system (1) in the discrete time scheme on the basis of some partition

$$
\Delta_{k}=\left\{\tau_{j}: \tau_{1}=t_{0}, \tau_{j}<\tau_{j+1}, j=\overline{1, k}, \tau_{k+1}=\vartheta\right\} .
$$

A triple $\left\{U(\cdot), \varepsilon, \Delta_{k}\right\}$ defines a control law, that forms a piecewise constant control realization according to the following step-by-step rule:

$$
u(t)=U\left(\tau_{j}, x\left(\tau_{j}\right), u_{\tau_{j}}(\cdot), \varepsilon\right), \quad \tau_{j} \leq t<\tau_{j+1}, \quad j=\overline{1, k},
$$

where $u_{\tau_{j}}(\cdot)$ is defined according to $(2)$, and

$$
u\left(\tau_{j}+\xi\right)=p_{0}\left(\tau_{j}+\xi-t_{0}\right), \quad t_{0}-\tau \leq \tau_{j}+\xi<t_{0} .
$$

By $\Omega=\Omega\left(U(\cdot), \varepsilon, \Delta_{k}\right)$ we denote the set of all triples $\left(x\left[t_{0}[\cdot] \vartheta\right], u\left[t_{0}[\cdot] \vartheta\right), v\left[t_{0}[\cdot] \vartheta\right)\right)$, where

- $v\left[t_{0}[\cdot] \vartheta\right)$ is an admissible disturbance realization,

- $u\left[t_{0}[\cdot] \vartheta\right)$ is the control realization formed by the control law $\left\{U(\cdot), \varepsilon, \Delta_{k}\right\}$,

- $x\left[t_{0}[\cdot] \vartheta\right]$ is the system motion generated by these realizations $u\left[t_{0}[\cdot] \vartheta\right)$ and $v\left[t_{0}[\cdot] \vartheta\right)$ from the initial position $\left(t_{0}, x_{0}, p_{0}(\cdot)\right)$.
The optimal guaranteed result $\Gamma^{0}$ is defined as follows:

$$
\Gamma^{0}=\inf _{U(\cdot)} \limsup _{\varepsilon \downarrow 0} \lim _{\delta \downarrow 0} \sup _{\Delta_{k}} \Gamma,
$$

where the last supremum is taken over all partitions $\Delta_{k}$ (4) with the diameter $\delta_{k}=\max _{j=\overline{1, k}}\left(\tau_{j+1}-\tau_{j}\right) \leq \delta$, and

$$
\Gamma=\sup \left\{\gamma:\left(x\left[t_{0}[\cdot] \vartheta\right], u\left[t_{0}[\cdot] \vartheta\right), v\left[t_{0}[\cdot] \vartheta\right)\right) \in \Omega\right\} .
$$

The problem under consideration is to find the optimal guaranteed result and to construct the corresponding control law that ensures this result.

\section{SOLUTION PROCEDURE}

Put

$$
h(t)=\min \left\{i=\overline{1, N}: \vartheta_{i} \geq t\right\}, \quad t \in\left[t_{0}, \vartheta\right],
$$

where instants $\vartheta_{i}$ are taken from cost function (3). By $h(t-0)$ and $h(t+0)$ we denote the left-hand and the righthand limits of the function $h(t)$ at the point $t \in\left[t_{0}, \vartheta\right]$, and in addition we put $h\left(t_{0}-0\right)=h\left(t_{0}\right), h(\vartheta+0)=h(\vartheta)$.

Let $X(t, \xi)$ be a fundamental solution matrix for the equation $\mathrm{d} x(t) / \mathrm{d} t=A(t) x(t)$, such that $X(\xi, \xi)=E$, where $E$ is the identity matrix. Define a $(2 n \times r)$-matrix $\mathbf{B}(t)$ and a $(2 n \times s)$-matrix $\mathbf{C}(t)$ by the following rule:

$$
\begin{aligned}
\mathbf{B}(t) & =\left(\begin{array}{c}
X\left(\vartheta_{h(t+0)}, t\right) B(t) \\
X(\vartheta, t)\left(B(t)+X(t, t+\tau) B_{\tau}(t+\tau) \chi(t)\right)
\end{array}\right), \\
\mathbf{C}(t) & =\left(\begin{array}{c}
X\left(\vartheta_{h(t+0)}, t\right) C(t) \\
X(\vartheta, t) C(t)
\end{array}\right),
\end{aligned}
$$

where $\chi(t)=1$ if $t<\vartheta-\tau$, and $\chi(t)=0$ if $t \geq \vartheta-\tau$.

Let $\Delta_{k}$ be the partition defined in (4). Here and everywhere below we assume that the partition $\Delta_{k}$ contains all the instants $\vartheta_{i}$ from the cost function:

$$
\vartheta_{i} \in \Delta_{k}, \quad i=\overline{1, N} \text {. }
$$

For every $j=\overline{1, k}$, denote

$\Delta \psi\left(\tau_{j}, g\right)=\int_{\tau_{j}}^{\tau_{j+1}} \max _{v \in Q} \min _{u \in P}\langle g, \mathbf{B}(t) u+\mathbf{C}(t) v\rangle \mathrm{d} t, \quad g \in \mathbb{R}^{2 n}$, where $\langle\cdot, \cdot\rangle$ stands for the inner product.

Everywhere below the notation $g=(l, m)$ means that the first $n$ coordinates of the vector $g \in \mathbb{R}^{2 n}$ coincide with the coordinates of the vector $l \in \mathbb{R}^{n}$, and the next $n$ coordinates of the vector $g \in \mathbb{R}^{2 n}$ coincide with the coordinates of the vector $m \in \mathbb{R}^{n}$.

Define sets $G\left(\tau_{j} \pm 0\right) \subset \mathbb{R}^{2 n}$ and functions $\varphi\left(\tau_{j} \pm 0, g\right)$, $g \in G\left(\tau_{j} \pm 0\right), j=\overline{1, k+1}$, by the following backward recurrence relations.

For $j=k+1$, put

$$
\begin{gathered}
G\left(\tau_{k+1}+0\right)=\{g=0\}, \\
G\left(\tau_{k+1}-0\right)=\left\{g=(l, m) \in \mathbb{R}^{2 n}:\|l\| \leq 1, m=0\right\}, \\
\varphi\left(\tau_{k+1} \pm 0, g\right)=0, \quad g \in G\left(\tau_{k+1} \pm 0\right) .
\end{gathered}
$$


For $j=\overline{1, k}$, put

$$
\begin{gathered}
G\left(\tau_{j}+0\right)=G\left(\tau_{j+1}-0\right), \\
\psi\left(\tau_{j}, g\right)=\Delta \psi\left(\tau_{j}, g\right)+\varphi\left(\tau_{j+1}-0, g\right), \quad g \in G\left(\tau_{j}+0\right), \\
\varphi\left(\tau_{j}+0, \cdot\right)=\left\{\psi\left(\tau_{j}, \cdot\right)\right\}_{G\left(\tau_{j}+0\right)}^{*},
\end{gathered}
$$

where $\{\psi(\cdot)\}_{G}^{*}$ denotes the upper convex hull of the function $\psi(\cdot)$ on the set $G$, i.e. the minimal concave function that majorizes $\psi(\cdot)$ on $G$. Further, if $\tau_{j} \neq \vartheta_{h}$, where $h=h\left(\tau_{j}\right)$, define

$$
G\left(\tau_{j}-0\right)=G\left(\tau_{j}+0\right),
$$

$$
\varphi\left(\tau_{j}-0, g\right)=\varphi\left(\tau_{j}+0, g\right), \quad g \in G\left(\tau_{j}-0\right),
$$

If $\tau_{j}=\vartheta_{h}$, put

$$
G\left(\tau_{j}-0\right)=\left\{g \in \mathbb{R}^{2 n}: M_{h}(g) \neq \emptyset\right\},
$$

$\varphi\left(\tau_{j}-0, g\right)=\max _{\left(\nu, g_{*}\right) \in M_{h}(g)} \nu \varphi\left(\tau_{j}+0, g_{*}\right), \quad g \in G\left(\tau_{j}-0\right)$,

where

$$
\begin{gathered}
M_{h}(g=(l, m))=\left\{\left(\nu, g_{*}=\left(l_{*}, m_{*}\right)\right) \in[0,1] \times G\left(\tau_{j}+0\right):\right. \\
\left.m=\nu\left(m_{*}+X^{T}\left(\vartheta_{h+1}, \vartheta\right) l_{*}\right), \nu^{2} \leq 1-\|l\|^{2}\right\} .
\end{gathered}
$$

Here and below the superscript $T$ denotes transposition.

Note that, for any $j=\overline{1, k+1}$, the sets $G\left(\tau_{j} \pm 0\right) \subset \mathbb{R}^{2 n}$ are convex and compact, the functions $\varphi\left(\tau_{j} \pm 0, g\right)$, $g \in G\left(\tau_{j} \pm 0\right)$, are concave, bounded and upper semicontinuous, and $0 \in G\left(\tau_{j} \pm 0\right), \varphi\left(\tau_{j} \pm 0,0\right) \geq 0$.

Put

$$
\begin{gathered}
e\left(\tau_{j} \pm 0, z\right)=\max _{g \in G\left(\tau_{j} \pm 0\right)}\left[\langle g, z\rangle+\varphi\left(\tau_{j} \pm 0, g\right)\right], \\
z \in \mathbb{R}^{2 n}, \quad j=\overline{1, k+1 .}
\end{gathered}
$$

Consider the following vectors $w_{1}=w_{1}(t \pm 0, x, p(\cdot)) \in \mathbb{R}^{n}$ and $w_{2}=w_{2}(t \pm 0, x, p(\cdot)) \in \mathbb{R}^{n}$ :

$$
\begin{aligned}
& w_{1}=X\left(\vartheta_{h(t \pm 0)}, t\right) x+\int_{t}^{\vartheta_{h(t \pm 0)}} X\left(\vartheta_{h(t \pm 0)}, \xi\right) B_{\tau}(\xi) p(\xi-t-\tau) d \xi \\
& w_{2}=X(\vartheta, t) x+\int_{t}^{t+\tau} X(\vartheta, \xi) B_{\tau}(\xi) \chi(\xi-\tau) p(\xi-t-\tau) d \xi .
\end{aligned}
$$

For $(t, x, p(\cdot)) \in K$, denote

$$
w(t \pm 0, x, p(\cdot))=\left(\begin{array}{c}
w_{1}(t \pm 0, x, p(\cdot)) \\
w_{2}(t \pm 0, x, p(\cdot))
\end{array}\right) \in \mathbb{R}^{2 n} .
$$

Let $j=\overline{1, k},\left(\tau_{j}, x, p(\cdot)\right) \in K$ and $\varepsilon>0$. Put

$$
z_{j}^{u}(x, p(\cdot), \varepsilon)=w\left(\tau_{j}+0, x, p(\cdot)\right)-\frac{m_{j}^{u} \sqrt{\varepsilon+\left(\tau_{j}-t_{0}\right) \varepsilon}}{\sqrt{1+\left\|m_{j}^{u}\right\|^{2}}},
$$

where

$$
\begin{aligned}
m_{j}^{u} & \in \underset{m \in G\left(\tau_{j}+0\right)}{\operatorname{argmax}}\left[\left\langle m, w\left(\tau_{j}+0, x, p(\cdot)\right)\right\rangle\right. \\
& \left.+\varphi\left(\tau_{j}+0, m\right)-\sqrt{\left(\varepsilon+\left(\tau_{j}-t_{0}\right) \varepsilon\right)\left(1+\|m\|^{2}\right)}\right] .
\end{aligned}
$$

Consider the following control strategy $U_{\Delta_{k}}(\cdot)$. For $t=$ $\tau_{j} \in \Delta_{k}$, this strategy is defined by the extremal shift to the accompanying point $z_{j}^{u}(\cdot)$ :

$$
\begin{gathered}
U_{\Delta_{k}}\left(\tau_{j}, x, p(\cdot), \varepsilon\right) \in \underset{u \in P}{\operatorname{argmin}}\left\langle s_{j}^{u}, \mathbf{B}\left(\tau_{j}\right) u\right\rangle, \\
\left(\tau_{j}, x, p(\cdot)\right) \in K, \quad j=\overline{1, k}
\end{gathered}
$$

where

$$
s_{j}^{u}=s_{j}^{u}(x, p(\cdot), \varepsilon)=w\left(\tau_{j}+0, x, p(\cdot)\right)-z_{j}^{u}(x, p(\cdot), \varepsilon) .
$$

For $t \neq \tau_{j} \in \Delta_{k}$, the strategy $U_{\Delta_{k}}(\cdot)$ is defined arbitrarily.

Theorem 1. For any number $\xi>0$, there exists a number $\delta>0$ such that, for any partition $\Delta_{k}(4)$, (7) with the diameter $\delta_{k} \leq \delta$, the following inequality holds

$$
\left|\Gamma^{0}-e\left(\tau_{1}-0, w\left(\tau_{1}-0, x_{0}, p_{0}(\cdot)\right)\right)\right| \leq \xi .
$$

Theorem 2. For any number $\zeta>0$, there exist a number $\varepsilon^{*}>0$ and a function $\delta(\varepsilon)>0, \varepsilon \in\left(0, \varepsilon^{*}\right]$, such that, for any value $\varepsilon \in\left(0, \varepsilon^{*}\right]$, and any partition $\Delta_{k}(4),(7)$ with the diameter $\delta_{k} \leq \delta(\varepsilon)$, the control law $\left\{U_{\Delta_{k}}(\cdot), \varepsilon, \Delta_{k}\right\}$ ensures the inequality

$$
\gamma \leq \Gamma^{0}+\zeta
$$

for any admissible disturbance realization $v\left[t_{0}[\cdot] \vartheta\right)$.

The proofs of Theorems 1 and 2 are given in Section 7 . In the next three sections we consider some auxiliary constructions.

\section{AUXILIARY $z$-MODEL}

Consider an auxiliary $z$-model described by the following differential equation

$$
\begin{gathered}
\frac{\mathrm{d} z(t)}{\mathrm{d} t}=\mathbf{B}(t) u_{*}(t)+\mathbf{C}(t) v_{*}(t), \\
t_{0} \leq t \leq \vartheta, \quad z \in \mathbb{R}^{2 n}, \quad u_{*} \in P, \quad v_{*} \in Q,
\end{gathered}
$$

where $z$ is the state vector, $u_{*}$ is the control vector, $v_{*}$ is the disturbance vector, matrix functions $\mathbf{B}(t)$ and $\mathbf{C}(t)$ are defined in (6).

Let numbers $\lambda_{w}>0$ and $\lambda_{z}>0$ be such that

$$
\begin{gathered}
\|w(t \pm 0, x, p(\cdot))\| \leq \lambda_{w}, \quad(t, x, p(\cdot)) \in K \\
\left\|\mathbf{B}(t) u_{*}+\mathbf{C}(t) v_{*}\right\| \leq \lambda_{z}, \quad\left(t, u_{*}, v_{*}\right) \in\left[t_{0}, \vartheta\right] \times P \times Q .
\end{gathered}
$$

A pair $(t, z)$ is called a position of $z$-model. The set $K_{z}$ of all possible positions is defined as follows

$K_{z}=\left\{(t, z) \in\left[t_{0}, \vartheta\right] \times \mathbb{R}^{2 n}:\|z\| \leq \lambda_{w}+1+\left(t-t_{0}\right) \lambda_{z}\right\}$. Let $\left(t_{*}, z_{*}\right) \in K_{z}, t_{*}<\vartheta$, and $t^{*} \in\left(t_{*}, \vartheta\right]$ be given. Admissible control and disturbance realizations are Borel measurable functions

$$
\begin{aligned}
& u_{*}\left[t_{*}[\cdot] t^{*}\right)=\left\{u_{*}(t) \in P, t_{*} \leq t<t^{*}\right\}, \\
& v_{*}\left[t_{*}[\cdot] t^{*}\right)=\left\{v_{*}(t) \in Q, t_{*} \leq t<t^{*}\right\} .
\end{aligned}
$$

From the position $\left(t_{*}, z_{*}\right)$ such realizations uniquely generate the motion

$$
z\left[t_{*}[\cdot] t^{*}\right]=\left\{z(t) \in \mathbb{R}^{2 n}, t_{*} \leq t \leq t^{*}\right\}
$$

that is an absolutely continuous function, which satisfies the condition $z\left(t_{*}\right)=z_{*}$ and, together with $u_{*}(t)$ and $v_{*}(t)$, satisfies equation (11) for almost all $t \in\left[t_{*}, t^{*}\right]$. Note that, for any $t \in\left[t_{*}, t^{*}\right]$, the inclusion $(t, z(t)) \in K_{z}$ is valid. 
Lemma 1. For any number $\varepsilon>0$, there exists a number $\delta>0$, such that the following holds. Let

- $\Delta_{k}$ be partition (4), (7) with the diameter $\delta_{k} \leq \delta$;

- $j=\overline{1, k},\left(\tau_{j}, x_{*}, p_{*}(\cdot)\right) \in K,\left(\tau_{j}, z_{*}\right) \in K_{z}$ and $s_{*}=w\left(\tau_{j}+0, x_{*}, p_{*}(\cdot)\right)-z_{*}$

- $x\left[\tau_{j}[\cdot] \tau_{j+1}\right]$ be a motion of system (1) generated from the position $\left(\tau_{j}, x_{*}, p_{*}(\cdot)\right)$ by some admissible disturbance realization $v\left[\tau_{j}[\cdot] \tau_{j+1}\right)$ and a constant control realization where

$$
u\left[\tau_{j}[\cdot] \tau_{j+1}\right)=\left\{u(t)=u^{\mathrm{e}}, \tau_{j} \leq t<\tau_{j+1}\right\},
$$

$$
u^{\mathrm{e}} \in \underset{u \in P}{\operatorname{argmin}}\left\langle s_{*}, \mathbf{B}\left(\tau_{j}\right) u\right\rangle ;
$$

- $z\left[\tau_{j}[\cdot] \tau_{j+1}\right]$ be a motion of $z$-model (11) generated from the position $\left(\tau_{j}, z_{*}\right)$ by some admissible control realization $u_{*}\left[\tau_{j}[\cdot] \tau_{j+1}\right)$ and a constant disturbance realization

$$
v_{*}\left[\tau_{j}[\cdot] \tau_{j+1}\right)=\left\{v_{*}(t)=v_{*}^{\mathrm{e}}, \tau_{j} \leq t<\tau_{j+1}\right\},
$$

where

$$
v_{*}^{\mathrm{e}} \in \underset{v_{*} \in Q}{\operatorname{argmax}}\left\langle s_{*}, \mathbf{C}\left(\tau_{j}\right) v_{*}\right\rangle .
$$

Then the following inequality is valid

$$
\begin{aligned}
\left\|w\left(\tau_{j+1}-0, x\left(\tau_{j+1}\right), u_{\tau_{j+1}}(\cdot)\right)-z\left(\tau_{j+1}\right)\right\|^{2} & \\
& \leq\left\|s_{*}\right\|^{2}+\left(\tau_{j+1}-\tau_{j}\right) \varepsilon .
\end{aligned}
$$

Here $u_{\tau_{j+1}}(\cdot)$ is defined according to $(2)$ and $u\left(\tau_{j+1}+\xi\right)=p_{*}\left(\tau_{j+1}+\xi-\tau_{j}\right), \quad \tau_{j}-\tau \leq \tau_{j+1}+\xi<\tau_{j}$.

Proof. We follow the scheme of the proof of Lemma 25.1 in Krasovskii (1985) (see also Section 2.3 in Krasovskii and Subbotin (1988)). Denote

$$
\begin{gathered}
s(t)=w\left(t+0, x(t), u_{t}(\cdot)\right)-z(t), \\
\Delta u(t)=u^{\mathrm{e}}-u_{*}(t), \quad \Delta v(t)=v(t)-v_{*}^{\mathrm{e}} .
\end{gathered}
$$

From (9), for almost all $t \in\left(\tau_{j}, \tau_{j+1}\right)$, we have

$$
\mathrm{d}\|s(t)\|^{2} / \mathrm{d} t=2\langle s(t), \mathbf{B}(t) \Delta u(t)+\mathbf{C}(t) \Delta v(t)\rangle .
$$

Due to continuity properties of $s(t)$ and $\mathbf{B}(t), \mathbf{C}(t)$ there exists such a function $\eta(\delta), \eta(\delta) \rightarrow 0$ when $\delta \rightarrow 0$, that the following estimation is valid

$$
\begin{aligned}
\langle s(t), \mathbf{B}(t) \Delta u(t)+\mathbf{C}(t) \Delta v(t)\rangle & \\
\leq & \left\langle s_{*}, \mathbf{B}\left(\tau_{j}\right) \Delta u(t)+\mathbf{C}\left(\tau_{j}\right) \Delta v(t)\right\rangle+\eta(\delta),
\end{aligned}
$$

whatever positions $\left(t, x(t), u_{t}(\cdot)\right) \in K$ and $(t, z(t)) \in K_{z}$, $\tau_{j} \leq t<\tau_{j+1} \leq \tau_{j}+\delta$. Using (12) and (13) we obtain

$$
\left\langle s_{*}, \mathbf{B}\left(\tau_{j}\right) \Delta u(t)+\mathbf{C}\left(\tau_{j}\right) \Delta v(t)\right\rangle \leq 0 .
$$

Hence, for almost all $t \in\left(\tau_{j}, \tau_{j+1}\right)$,

$$
\mathrm{d}\|s(t)\|^{2} / \mathrm{d} t \leq 2 \eta(\delta) .
$$

Integrating this inequality, by (9) we get

$$
\begin{aligned}
\left\|w\left(\tau_{j+1}-0, x\left(\tau_{j+1}\right), u_{\tau_{j+1}}(\cdot)\right)-z\left(\tau_{j+1}\right)\right\|^{2} & \\
& \leq\left\|s_{*}\right\|^{2}+2\left(\tau_{j+1}-\tau_{j}\right) \eta(\delta) .
\end{aligned}
$$

Thus, inequality (14) is fulfilled if we choose $\delta>0$ such that $\eta(\delta) \leq \varepsilon / 2$.

\section{PROPERTIES OF THE VALUES $e\left(\tau_{j} \pm 0, \cdot\right)$.}

Let $\Delta_{k}$ be partition (4), (7) and values $e\left(\tau_{j} \pm 0, \cdot\right), j=$ $\overline{1, k+1}$, be defined by (8) on the basis of this partition.
The following Lemma establishes the $u$-stability property (see, e.g., Krasovskii and Krasovskii (1995); Lukoyanov (1998)) of these values with respect to $z$-model (11).

Lemma 2. Let $j=\overline{1, k},\left(\tau_{j}, z_{*}\right) \in K_{z}$ and $v_{*} \in Q$. Then, for the disturbance realization

$$
v_{*}\left[\tau_{j}[\cdot] \tau_{j+1}\right)=\left\{v_{*}(t)=v_{*}, \tau_{j} \leq t<\tau_{j+1}\right\},
$$

there exists an admissible control realization $u_{*}\left[\tau_{j}[\cdot] \tau_{j+1}\right)$ such that, for the motion $z\left[\tau_{j}[\cdot] \tau_{j+1}\right]$ of $z$-model generated by these realizations from the position $\left(\tau_{j}, z_{*}\right)$, the following inequality holds

$$
e\left(\tau_{j}+0, z_{*}\right) \geq e\left(\tau_{j+1}-0, z\left(\tau_{j+1}\right)\right) .
$$

The proof of this Lemma is similar to the proof of the $u$-stability property in Lukoyanov (1998).

To prove Theorems 1 and 2 we also need the next result. Lemma 3. Let $j=\overline{1, k}$ and $h=h\left(\tau_{j}\right)$ be defined by (5). If $\tau_{j} \neq \vartheta_{h}$, then, for any $z \in \mathbb{R}^{2 n}$,

$$
e\left(\tau_{j}-0, z\right)=e\left(\tau_{j}+0, z\right),
$$

If $\tau_{j} \neq \vartheta_{h}$, then, for any $x \in \mathbb{R}^{n}$ and $p(\cdot) \in \mathcal{P}$,

$$
e^{2}\left(\tau_{j}-0, w_{j}^{-}\right)=\|x\|^{2}+e^{2}\left(\tau_{j}+0, w_{j}^{+}\right) .
$$

Here the vectors $w_{j}^{ \pm}=w\left(\tau_{j} \pm 0, x, p(\cdot)\right)$ are defined by $(9)$.

This Lemma is proved similarly to Lemma 1 in Lukoyanov (1998).

\section{PROPERTIES OF THE STRATEGY $U_{\Delta_{k}}(\cdot)$}

Consider the strategy $U_{\Delta_{k}}(\cdot)$ defined by (10).

Lemma 4. For any number $\zeta>0$, there exist a number $\varepsilon^{*}>0$ and a function $\delta(\varepsilon)>0, \varepsilon \in\left(0, \varepsilon^{*}\right]$, such that, for any value $\varepsilon \in\left(0, \varepsilon^{*}\right]$, and any partition $\Delta_{k}(4),(7)$ with the diameter $\delta_{k} \leq \delta(\varepsilon)$, the control law $\left\{U_{\Delta_{k}}(\cdot), \varepsilon, \Delta_{k}\right\}$ ensures the inequality

$$
\gamma \leq e\left(\tau_{1}-0, w\left(\tau_{1}-0, x_{0}, p_{0}(\cdot)\right)\right)+\zeta
$$

for any admissible disturbance realization $v\left[t_{0}[\cdot] \vartheta\right)$.

Proof. Let a number $L>0$ be such that, for any $j=$ $\overline{1, k+1}$ and any $z_{1}, z_{2} \in \mathbb{R}^{2 n}$,

$$
\left|e\left(\tau_{j}-0, z_{1}\right)-e\left(\tau_{j}-0, z_{2}\right)\right| \leq L\left\|z_{1}-z_{2}\right\|,
$$

and $L$ do not depend on the partition $\Delta_{k}$.

Choose $\varepsilon^{*}>0$ such that

$$
\sqrt{\varepsilon^{*}+\varepsilon^{*}\left(\vartheta-t_{0}\right)} \leq \min \{1, \zeta /(N(L+1))\},
$$

where $N$ is the number of instants $\vartheta_{i}$ from cost function (3). For every $\varepsilon \in\left(0, \varepsilon^{*}\right]$, take $\delta=\delta(\varepsilon)>0$ from Lemma 1. Let us show that such $\varepsilon^{*}$ and $\delta(\varepsilon)$ satisfy this Lemma.

Let $x\left[t_{0}[\cdot] \vartheta\right]$ be a motion of system (1) generated from the initial position $\left(t_{0}, x_{0}, p_{0}(\cdot)\right)$ by the control law $\left\{U_{\Delta_{k}}(\cdot), \varepsilon, \Delta_{k}\right\}$ in a pair with some admissible disturbance realization $v\left[t_{0}[\cdot] \vartheta\right)$. Let $u\left[t_{0}[\cdot] \vartheta\right)$ be the corresponding control realization, and $u(t)=p_{0}\left(t-t_{0}\right)$ when $t_{0}-\tau \leq t<t_{0}$. Denote

$$
\begin{aligned}
w_{j}^{ \pm} & =w\left(\tau_{j} \pm 0, x\left(\tau_{j}\right), u_{\tau_{j}}(\cdot)\right), \quad j=\overline{1, k+1}, \\
z_{j}^{u} & =z_{j}^{u}\left(x\left(\tau_{j}\right), u_{\tau_{j}}(\cdot), \varepsilon\right), \\
\alpha_{j} & =-\frac{\sqrt{\varepsilon+\left(\tau_{j}-t_{0}\right) \varepsilon}}{\sqrt{1+\left\|m_{j}^{u}\left(x\left(\tau_{j}\right), u_{\tau_{j}}(\cdot), \varepsilon\right)\right\|^{2}}}, \quad j=\overline{1, k} .
\end{aligned}
$$


Note that, for any $j=\overline{1, k}$, we have $\left(\tau_{j}, z_{j}^{u}\right) \in K_{z}$, and (see, e.g., Kornev (2012))

$$
\left(z_{j}^{u}, \alpha_{j}\right) \in \operatorname{argmin}\left[e\left(\tau_{j}+0, z\right)+\alpha\right],
$$

where minimum is taken over all pairs $(z, \alpha)$ such that

$$
\left\|w_{j}^{+}-z\right\|^{2}+\alpha^{2} \leq \varepsilon+\left(\tau_{j}-t_{0}\right) \varepsilon .
$$

Fix $j=\overline{1, k}$, denote $s_{*}=w_{j}^{+}-z_{j}^{u}$, and choose $v_{*}^{\mathrm{e}} \in Q$ by (13). For the constant disturbance realization

$$
v_{*}\left[\tau_{j}[\cdot] \tau_{j+1}\right)=\left\{v_{*}(t)=v_{*}^{\mathrm{e}}, \tau_{j} \leq t<\tau_{j+1}\right\},
$$

take $u_{*}\left[\tau_{j}[\cdot] \tau_{j+1}\right)$ according to Lemma 2 and consider the corresponding motion $z^{(j)}\left[\tau_{j}[\cdot] \tau_{j+1}\right]$ of $z$-model (11) generated from the position $\left(\tau_{j}, z_{j}^{u}\right)$. Then, by Lemma 2 ,

$$
e\left(\tau_{j}+0, z_{j}^{u}\right) \geq e\left(\tau_{j+1}-0, z^{(j)}\left(\tau_{j+1}\right)\right)
$$

and, by Lemma 1 , if we take (21), (10), and (12) into account,

$$
\left\|w_{j+1}^{-}-z^{(j)}\left(\tau_{j+1}\right)\right\|^{2}+\alpha_{j}^{2} \leq \varepsilon+\left(\tau_{j+1}-t_{0}\right) \varepsilon .
$$

Let us prove, for each $j=\overline{1, k}$, the inequality

$$
e\left(\tau_{j}+0, z_{j}^{u}\right)+\alpha_{j} \geq \sqrt{\sum_{i=h\left(\tau_{j}+0\right)}^{N}\left\|x\left(\vartheta_{i}\right)\right\|^{2}}-\beta_{j},
$$

where

$$
\beta_{j}=\left(N-h\left(\tau_{j}+0\right)+1\right)(L+1) \sqrt{\varepsilon+\left(\vartheta-t_{0}\right) \varepsilon} .
$$

Consider the induction on $j$ from $k$ to 1 .

Let $j=k$. From (8) and (9), we have

$$
e\left(\tau_{k+1}-0, w_{k+1}^{-}\right)=\left\|x\left(\vartheta_{N}\right)\right\| .
$$

Then, by (22), (18) and (23), we get (24) for $j=k$.

Now, we assume that inequality (24) is valid for $j=q$, $1<q \leq k$, and prove it for $j=q-1$.

If $\tau_{q} \neq \vartheta_{h}, h=h\left(\tau_{q}\right)$, according to (9) we have $w_{q}^{+}=w_{q}^{-}$. So, by (23), we obtain

$$
\left\|w_{q}^{+}-z^{(q-1)}\left(\tau_{q}\right)\right\|^{2}+\alpha_{q-1}^{2} \leq \varepsilon+\left(\tau_{q}-t_{0}\right) \varepsilon .
$$

Thus, from (15) and (20), we get

$$
e\left(\tau_{q}-0, z^{(q-1)}\left(\tau_{q}\right)\right)+\alpha_{q-1} \geq e\left(\tau_{q}+0, z_{q}^{u}\right)+\alpha_{q} .
$$

So, in this case, inequality (24) is valid for $j=q-1$ due to the induction hypothesis, equality $h\left(\tau_{q-1}+0\right)=h\left(\tau_{q}+0\right)$ and inequality (22).

If $\tau_{q}=\vartheta_{h}$, according to $(20)-(22)$, by the induction hypothesis, we have

$e\left(\tau_{q}+0, w_{q}^{+}\right) \geq e\left(\tau_{q}+0, z_{q}^{u}\right)+\alpha_{q} \geq \sqrt{\sum_{i=h\left(\tau_{q}+0\right)}^{N}\left\|x\left(\vartheta_{i}\right)\right\|^{2}}-\beta_{q}$.

Then, using equalities $(16)$ and $h\left(\tau_{q-1}+0\right)=h\left(\tau_{q}+0\right)-1$, we obtain

$$
e\left(\tau_{q}-0, w_{q}^{-}\right) \geq \sqrt{\sum_{i=h\left(\tau_{q-1}+0\right)}^{N}\left\|x\left(\vartheta_{i}\right)\right\|^{2}}-\beta_{q} .
$$

Therefore, in this case, the validity of (24) for $j=q-1$ follows from inequalities (22), (18) and (23).
Using (20) and (24) for $j=1$, by (19), we deduce

$e\left(\tau_{1}+0, w_{1}^{+}\right) \geq e\left(\tau_{1}+0, z_{1}^{u}\right)+\alpha_{1} \geq \sqrt{\sum_{i=h\left(\tau_{1}+0\right)}^{N}\left\|x\left(\vartheta_{i}\right)\right\|^{2}}-\zeta$.

Hence, accoeding to (15), we conclude, that, for the realized value $\gamma$ of cost function (3), inequality (17) holds.

Besides the control law $\left\{U_{\Delta_{k}}(\cdot), \varepsilon, \Delta_{k}\right\}$, we consider also a disturbance law $\left\{V_{\Delta_{k}}(\cdot), \varepsilon, \Delta_{k}\right\}$ that forms a piecewise constant disturbance realization as follows

$$
\begin{gathered}
v(t)=V_{\Delta_{k}}\left(\tau_{j}, x\left(\tau_{j}\right), u_{\tau_{j}}(\cdot), \varepsilon\right) \in \underset{v \in Q}{\operatorname{argmax}}\left\langle s_{j}^{v}, \mathbf{C}\left(\tau_{j}\right) v\right\rangle, \\
\tau_{j} \leq t<\tau_{j+1}, \quad j=\overline{1, k}
\end{gathered}
$$

where

$$
\begin{aligned}
s_{j}^{v} & =\frac{m_{j}^{v} \sqrt{\varepsilon+\left(\tau_{j}-t_{0}\right) \varepsilon}}{\sqrt{1+\left\|m_{j}^{v}\right\|^{2}}}, \\
m_{j}^{v} & \in \underset{m \in G\left(\tau_{j}+0\right)}{\operatorname{argmax}}\left[\left\langle m, w\left(\tau_{j}+0, x\left(\tau_{j}\right), u_{\tau_{j}}(\cdot)\right)\right\rangle\right. \\
& \left.+\varphi\left(\tau_{j}+0, m\right)+\sqrt{\left(\varepsilon+\left(\tau_{j}-t_{0}\right) \varepsilon\right)\left(1+\|m\|^{2}\right)}\right] .
\end{aligned}
$$

Here, as usual, $u_{\tau_{j}}(\cdot)$ is defined by $(2)$ and

$$
u\left(\tau_{j}+\xi\right)=p_{0}\left(\tau_{j}+\xi-t_{0}\right), \quad t_{0}-\tau \leq \tau_{j}+\xi<t_{0} .
$$

Lemma 5. For any number $\zeta>0$, there exist a number $\varepsilon^{*}>0$ and a function $\delta(\varepsilon)>0, \varepsilon \in\left(0, \varepsilon^{*}\right]$, such that, for any value $\varepsilon \in\left(0, \varepsilon^{*}\right]$, and any partition $\Delta_{k}(4),(7)$ with the diameter $\delta_{k} \leq \delta(\varepsilon)$, the disturbance law $\left\{V_{\Delta_{k}}(\cdot), \varepsilon, \Delta_{k}\right\}$ ensures the inequality

$$
\gamma \geq e\left(\tau_{1}-0, w\left(\tau_{1}-0, x_{0}, p_{0}(\cdot)\right)\right)-\zeta
$$

for any admissible control realization $v\left[t_{0}[\cdot] \vartheta\right)$.

This Lemma is proved similarly to Lemma 5 by using the corresponding modification of Lemma 1 and the appropriate $v$-stability property (see Lukoyanov (1998)) of values (8) with respect to $z$-model (11).

\section{PROOFS OF THEOREMS 1 AND 2}

Theorem 2 follows directly from Lemma 4 and Theorem 1. Let us prove Theorem 1 .

As was shown in Gomoyunov and Lukoyanov (2012), for the control problem (1), (3), there exist the optimal control strategy $U^{0}(\cdot)$ and the counter-optimal disturbance strategy $V^{0}(\cdot)$. In particular, it means that, for the number $\zeta=\xi / 2>0$, there exist a number $\varepsilon_{0}^{*}>0$ and a function $\delta_{0}(\varepsilon)>0, \varepsilon \in\left(0, \varepsilon_{0}^{*}\right]$, such that, for any value $\varepsilon \in\left(0, \varepsilon_{0}^{*}\right]$, and any partition $\Delta_{k}$ (4) with the diameter $\delta_{k} \leq \delta_{0}(\varepsilon)$, on the one hand, the control law $\left\{U^{0}(\cdot), \varepsilon, \Delta_{k}\right\}$ ensures the inequality

$$
\gamma \leq \Gamma^{0}+\zeta
$$

for any admissible disturbance realization $v\left[t_{0}[\cdot] \vartheta\right)$, and on the other hand, the disturbance law $\left\{V^{0}(\cdot), \varepsilon, \Delta_{k}\right\}$, which forms the piecewise constant disturbance realization $v(t)=V^{0}\left(\tau_{j}, x\left(\tau_{j}\right), u_{\tau_{j}}(\cdot), \varepsilon\right), \quad \tau_{j} \leq t<\tau_{j+1}, \quad j=\overline{1, k}$, ensures the inequality

$$
\gamma \geq \Gamma^{0}-\zeta
$$

for any admissible control realization $u\left[t_{0}[\cdot] \vartheta\right)$. 
In order to ensure inequalities (17) and (25) for $\zeta=\xi / 2$, take $\varepsilon_{1}^{*}>0, \varepsilon_{2}^{*}>0$ and $\delta_{1}(\varepsilon)>0, \delta_{2}(\varepsilon)>0$ by using Lemmas 4 and 5. Put

$\varepsilon=\min \left\{\varepsilon_{0}^{*}, \varepsilon_{1}^{*}, \varepsilon_{2}^{*}\right\}>0, \quad \delta=\min \left\{\delta_{0}(\varepsilon), \delta_{1}(\varepsilon), \delta_{2}(\varepsilon)\right\}>0$. Let us show that this $\delta$ satisfies Theorem 1 .

Consider the motion of system (1) generated from the initial position $\left(t_{0}, x_{0}, p_{0}(\cdot)\right)$ by the laws $\left\{U_{\Delta_{k}}(\cdot), \varepsilon, \Delta_{k}\right\}$ and $\left\{V^{0}(\cdot), \varepsilon, \Delta_{k}\right\}$. Then, due to (17) and (27), we obtain $\Gamma^{0}-\zeta \leq \gamma \leq e\left(\tau_{1}-0, w\left(\tau_{1}-0, x_{0}, p_{0}(\cdot)\right)\right)+\zeta$,

and, consequently,

$$
\Gamma^{0}-e\left(\tau_{1}-0, w\left(\tau_{1}-0, x_{0}, p_{0}(\cdot)\right)\right) \leq 2 \zeta=\xi .
$$

Similarly, considering the motion of system (1) generated from the initial position $\left(t_{0}, x_{0}, p_{0}(\cdot)\right)$ by the control law $\left\{U^{0}(\cdot), \varepsilon, \Delta_{k}\right\}$ and the disturbance law $\left\{V_{\Delta_{k}}(\cdot), \varepsilon, \Delta_{k}\right\}$, due to (25) and (26), we have

$$
\Gamma^{0}-e\left(\tau_{1}-0, w\left(\tau_{1}-0, x_{0}, p_{0}(\cdot)\right)\right) \geq-2 \zeta=-\xi .
$$

Inequalities (28) and (29) complete the proof.

\section{EXAMPLE}

Consider a dynamical system described by the following differential equation

$$
\begin{aligned}
\frac{\mathrm{d} x(t)}{\mathrm{d} t}=\sin ( & (t+1)) x(t) \\
& +(1-0.1 t) u(t)+0.1 t u(t-1)+0.8 v(t),
\end{aligned}
$$

$0 \leq t \leq 10, \quad x \in \mathbb{R}, \quad u \in[-1,1], \quad v \in[-1,1]$,

and the initial condition

$$
x_{0}=1, \quad p_{0}(\xi)=0, \quad \xi \in[-1,0) .
$$

The motion quality is evaluated by the cost function

$$
\gamma=\left(\sum_{i=1}^{10} x^{2}(i)\right)^{1 / 2}
$$

Note that, in control problem (30)-(32), for a solution procedure from Gomoyunov and Lukoyanov (2012), the dimension of the domains of the auxiliary functions being convexified is equal to 10, whereas, for the procedure described in Section 3, this dimension is equal to 2 .

Let $\Delta_{k}$ be a uniform partition of the control interval $[0,10]$ with the diameter $\delta_{k}=0.005$ and $\varepsilon=0.05$. By using the solution procedure (Section 3 ) we numerically calculate the values $e\left(\tau_{j} \pm 0, \cdot\right), j=\overline{1, k+1}$, and construct the control law $\left\{U_{\Delta_{k}}(\cdot), \varepsilon, \Delta_{k}\right\}$. For related techniques of the software implementation see Kornev (2012).

The obtained a priori calculated value of the optimal guaranteed result is

$$
\Gamma^{0} \approx e\left(\tau_{1}-0, w\left(\tau_{1}-0, x_{0}, p_{0}(\cdot)\right)\right) \approx 1.808 .
$$

The results of the numerical simulations are shown in Fig. 1. We consider the following motions $x^{(i)}[0[\cdot] 10], i=$ $\overline{1,4}$, of system (30) generated from initial position (31).

The motion $x^{(1)}[0[\cdot] 10]$ (green line) is generated by the laws $\left\{U_{\Delta_{k}}(\cdot), \varepsilon, \Delta_{k}\right\}$ and $\left\{V_{\Delta_{k}}(\cdot), \varepsilon, \Delta_{k}\right\}$.

The motion $x^{(2)}[0[\cdot] 10]$ (orange line) is generated by the control law $\left\{U_{\Delta_{k}}(\cdot), \varepsilon, \Delta_{k}\right\}$ and disturbance actions:

$$
v(t)=\operatorname{sign}\left(x\left(\tau_{j}\right)\right), \quad \tau_{j} \leq t<\tau_{j+1}, \quad j=\overline{1, k} .
$$

The motion $x^{(3)}[0[\cdot] 10]$ (red line) is generated by the control law $\left\{U_{\Delta_{k}}(\cdot), \varepsilon, \Delta_{k}\right\}$ and zero disturbances.

The motion $x^{(4)}[0[\cdot] 10]$ (blue line) is generated by the disturbance law $\left\{V_{\Delta_{k}}(\cdot), \varepsilon, \Delta_{k}\right\}$ and control actions:

$$
u(t)=-\operatorname{sign}\left(x\left(\tau_{j}\right)\right), \quad \tau_{j} \leq t<\tau_{j+1}, \quad j=\overline{1, k} .
$$

The corresponding values of cost function (32) are

$$
\begin{aligned}
& \gamma^{(1)}=1.791 \approx \Gamma^{0}, \quad \gamma^{(2)}=1.791 \approx \Gamma^{0}, \\
& \gamma^{(3)}=0.03<\Gamma^{0}, \quad \gamma^{(4)}=2.536>\Gamma^{0} .
\end{aligned}
$$

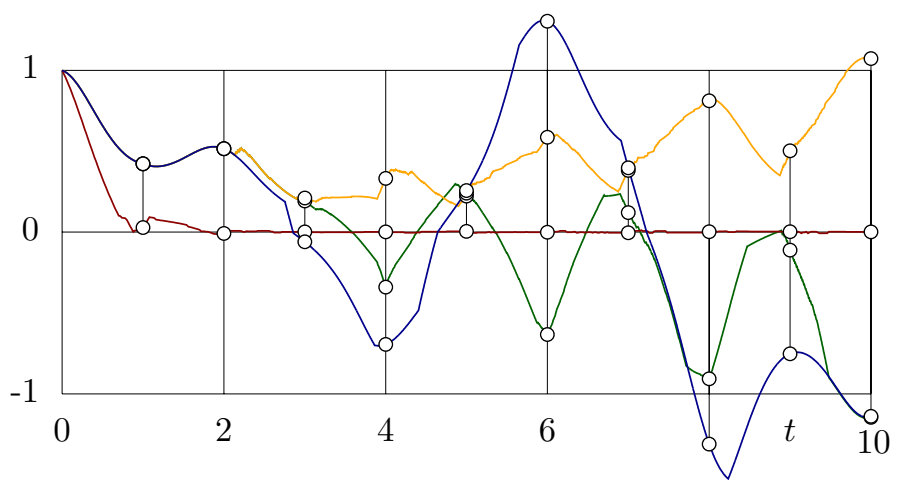

Fig. 1. The results of the numerical simulations in control problem (30)-(32).

\section{REFERENCES}

N.N. Krasovskii and A.I. Subbotin. Game-Theoretical Control Problems. Springer-Verlag, New York, 1988.

N.N. Krasovskii. Control of a dynamical system. Nauka, Moscow, 1985. (in Russian)

A.N. Krasovskii and N.N. Krasovskii. Control under lack of information. Birkhäuser, Berlin, 1995.

N.Yu. Lukoyanov. The problem of computing the value of a differential game for a positional functional. Journal of Applied Mathematics and Mechanics, volume 62, No. 2, pages 177-186, 1998.

Yu.S. Osipov and V.G. Pimenov. On positional control under aftereffect in the controlling forces. Journal of Applied Mathematics and Mechanics, volume 45, No. 2, pages 159-163, 1981.

H.T. Banks, M.Q. Jakobs and M.R. Latina. The synthesis of optimal controls for linear time-optimal problems with retarded controls. Journal of Optimization Theory and Applications, volume 8, No. 5, pages 319-366, 1971.

M.I. Gomoyunov and N.Yu. Lukoyanov. Dynamical optimization of systems with control delays. 15th IFAC Workshop on Control Applications of Optimization, Rimini, Italy, volume 15, No. 1, pages 100-105, 2012. (URL:http://www.ifacpapersonline.net/Detailed/56631.html)

D.V. Kornev. On numerical solution of positional differential games with nonterminal payoff. Automation and Remote Control, volume 73, No. 11, pages 1808-1821, 2012. 